\title{
Radiative corrections to the triple Higgs coupling in the inert Higgs doublet model
}

\author{
Abdesslam Arhrib, ${ }^{a, b}$ Rachid Benbrik, $^{c, d}$ Jaouad El Falaki $^{a}$ and Adil Jueid ${ }^{a}$ \\ ${ }^{a}$ Département de Mathématiques, Faculté des Sciences et Techniques, \\ Université Abdelmalek Essaadi, B. 416, Tangier, Morocco \\ ${ }^{b}$ Physics Division, National Center for Theoretical Sciences, \\ Hsinchu, 300 Taiwan \\ ${ }^{c}$ MSISM team, Faculty of Polydisciplinaire de Safi, University Cadi Ayyad Sidi Bouzid, \\ B.P. 4162, Safi, 46000 Morocco \\ ${ }^{d}$ LPHEA, Faculty of Science Semlalia, Cadi Ayyad University, \\ Marrakesh, Morocco \\ E-mail: aarhrib@ictp.it, rbenbrik@ictp.it, jaouad.elfalaki@gmail.com, \\ ajueid@ictp.it
}

ABSTRACT: We investigate the implication of the recent discovery of a Higgs-like particle in the first phase of the LHC Run 1 on the Inert Higgs Doublet Model (IHDM). The determination of the Higgs couplings to SM particles and its intrinsic properties will get improved during the new LHC Run 2 starting this year. The new LHC Run 2 would also shade some light on the triple Higgs coupling. Such measurement is very important in order to establish the details of the electroweak symmetry breaking mechanism. Given the importance of the Higgs couplings both at the LHC and $e^{+} e^{-}$Linear Collider machines, accurate theoretical predictions are required. We study the radiative corrections to the triple Higgs coupling $h h h$ and to $h Z Z, h W W$ couplings in the context of the IHDM. By combining several theoretical and experimental constraints on parameter space, we show that extra particles might modify the triple Higgs coupling near threshold regions. Finally, we discuss the effect of these corrections on the double Higgs production signal at the $e^{+} e^{-}$ LC and show that they can be rather important.

KeYwords: Higgs Physics, Standard Model

ARXIV EPRINT: 1507.03630 


\section{Contents}

1 Introduction 1

2 The inert Higgs doublet model 2

2.1 The model 2

2.2 Theoretical and experimental constraints 3

3 Radiative corrections to triple Higgs couplings $h h h$ and $h Z Z \quad 5$

4 Numerical results $\quad 8$

4.1 SM case 8

4.2 IHDM case 8

5 Radiative corrections to $e^{+} e^{-} \rightarrow h h Z$ in the IHDM $\quad 12$

$\begin{array}{lll}5.1 & e^{+} e^{-} \rightarrow h h Z \text { in SM } & 12\end{array}$

5.2 One-loop corrections to $e^{+} e^{-} \rightarrow Z h h \quad 13$

6 Conclusion $\quad 15$

A The triple Higgs coupling in the IHDM: analytical results 16

\section{Introduction}

The discovery of a new particle with a mass around $125-126 \mathrm{GeV}$ in the search for the Standard Model (SM) Higgs boson [1-4] was announced simultaneously by the ATLAS and CMS collaborations in July 2012 [5-10]. Since then, more data has been taken and analyzed at the LHC. One of the primary goals of the Higgs groups at the LHC is now to study the properties of this new resonance and determine if it is indeed the state predicted by the SM.

With this new discovery, a program of precision measurement involving the Higgs boson has just started and will get improved with the new run of LHC and future $e^{+} e^{-}$Linear Collider (LC). In fact, the $7 \oplus 8 \mathrm{TeV}$ data allow the measurement of the Higgs couplings to gauge bosons and $\tau^{+} \tau^{-}$with about $20-30 \%$ of precision while the Higgs couplings to $b \bar{b}$ and $t \bar{t}$ still suffer large uncertainties of about $40-50 \%$. All these measurements will be improved with the new run of LHC at $13-14 \mathrm{TeV}$ and the future $e^{+} e^{-} \mathrm{LC}$.

In order to confirm that the discovered Higgs-like particle is the SM Higgs responsible for the electroweak symmetry breaking, we need to know all its couplings to SM particles with accurate precision and also measure the trilinear and quartic self-couplings of the Higgs in order to be able to reconstruct the scalar potential. In this regards, the LHC with its high luminosity option have also the capability of measuring the SM triple Higgs 
couplings through one of the following channels $g g \rightarrow h h \rightarrow b \bar{b} \gamma \gamma, b \bar{b} \tau^{+} \tau^{-}, b \bar{b} W^{ \pm} W^{\mp *}[11-$ 20]. The $e^{+} e^{-}$LC, which will provide some high precision measurement of the Higgs mass and its properties such as couplings to SM particles and quantum numbers, would also be able to perform SM triple Higgs coupling through $e^{+} e^{-} \rightarrow Z h h$ (double Higgs-Strahlung) and $e^{+} e^{-} \rightarrow \nu_{e} \bar{\nu}_{e} h h$ (WW fusion) with more than $700 \mathrm{GeV}$ center of mass energy with better precision [21-24]. In the double Higgs-Strahlung process $Z h h$ the $\mathrm{Z}$ boson will be reconstructed from $l^{+} l^{-}$or $q \bar{q}$ pairs, while for the WW fusion process the two Higgs can be reconstructed from $b \bar{b} b \bar{b}$ or $b \bar{b}$ and $W^{+} W^{-}$.

The discovery of this Higgs-like particle resonance opens a new era in elementary particle physics and leads to several theoretical and phenomenological studies on Higgs physics both in the SM and beyond. One of the very simplest extension of SM is the IHDM proposed, more than three decade ago, by Deshpande and Ma [25] for electroweak symmetry breaking purpose. Recently, the IHDM model has been very attractive because it provides a dark matter candidate [26-30, 32], generates tiny neutrino masses [33, 34] and also solves the naturalness problem [35] finally a dynamical realization of the IHDM by means of UV completion was studied in [31]. Phenomenology of IHDM have been extensively studied during last decade [32, 36, 37].

The aim of this paper is to study the effect of the one loop radiative corrections to the triple Higgs coupling $h h h$ as well as $h Z Z$ coupling in the framework of the IHDM. We will also compute the well known SM radiative corrections to the triple Higgs coupling as check of our procedure. Once these effects are well studied, we then proceed to the evaluation of radiative corrections to the double Higgs Strahlung process $e^{+} e^{-} \rightarrow Z h h$. For this purpose, we will apply an on-shell renormalization scheme to evaluate these one-loop corrections. For the numerical evaluation, we will take into account all the theoretical and experimental constraints on the scalar sector of the Model.

The paper is organized as follow: in the second section we introduce the IHDM model and describe the theoretical and experimental constraints that the model is subject to. In the third section we introduce the on-shell renormalization scheme for the triple Higgs coupling $h h h$ and $h Z Z$ in the IHDM and present our numerical results in the fourth section. Numerical analysis of the double Higgs-Strahlung is presented in the fifth section. Our conclusion is given in the last section.

\section{The inert Higgs doublet model}

\section{$2.1 \quad$ The model}

The IHDM is one of the most simplest models for the scalar dark matter, a version of a two Higgs double model with an exact $Z_{2}$ symmetry. The SM scalar sector is extended by an inert scalar doublet $H_{2}$ which can provide a stable dark matter candidate. Under $Z_{2}$ symmetry all the SM particles are even while $H_{2}$ is odd and it could mix with the SM-like Higgs doublet. We shall use the following parameterization of the two doublets:

$$
H_{1}=\left(\begin{array}{c}
G^{ \pm} \\
\frac{1}{\sqrt{2}}\left(v+h+i G^{0}\right)
\end{array}\right), \quad H_{2}=\left(\begin{array}{c}
H^{ \pm} \\
\frac{1}{\sqrt{2}}\left(H^{0}+i A^{0}\right)
\end{array}\right)
$$


with $G^{0}$ and $G^{ \pm}$are the Nambu-Goldstone bosons absorbed by the longitudinal component of $W^{ \pm}$and $Z^{0}$,respectively. $v$ is the vacuum expectation value (VEV) of the SM Higgs $H_{1}$. Within the IHDM the scalar doublet $H_{2}$ does not couple with the SM fermions and therefore the $\mathrm{H}_{2}$-fermions interaction are present only through mixing with $H_{1}$. The most general renormalizable, gauge invariant and $\mathrm{CP}$ invariant potential is given by:

$$
\begin{aligned}
V= & \mu_{1}^{2}\left|H_{1}\right|^{2}+\mu_{2}^{2}\left|H_{2}\right|^{2}+\lambda_{1}\left|H_{1}\right|^{4}+\lambda_{2}\left|H_{2}\right|^{4}+\lambda_{3}\left|H_{1}\right|^{2}\left|H_{2}\right|^{2}+\lambda_{4}\left|H_{1}^{\dagger} H_{2}\right|^{2} \\
& +\frac{\lambda_{5}}{2}\left\{\left(H_{1}^{\dagger} H_{2}\right)^{2}+\text { h.c }\right\} .
\end{aligned}
$$

In the above potential there is no mixing terms like $\mu_{12}^{2}\left(H_{1}^{\dagger} H_{2}+\right.$ h.c. $)$ because of the unbroken $Z_{2}$ symmetry. By hermicity of the potential, all $\lambda_{i}, i=1, \cdots, 4$ parameters are real. The phase of $\lambda_{5}$ can be absorbed by a suitable redefinition of the fields $H_{1}$ and $H_{2}$, therefore the scalar sector is CP conserving. After spontaneous symmetry breaking of $\mathrm{SU}(2)_{L} \otimes \mathrm{U}(1)_{Y}$ down to $\mathrm{U}(1)_{Q}$, the spectrum of this potential will have five scalar particles: two CP even $H^{0}$ and $h$ which will be identified as the SM Higgs boson, a CP odd $A^{0}$ and a pair of charged scalars $H^{ \pm}$. Their masses are given by:

$$
\begin{aligned}
m_{h}^{2} & =-2 \mu_{1}^{2}=2 \lambda_{1} v^{2} \\
m_{H^{0}}^{2} & =\mu_{2}^{2}+\lambda_{L} v^{2} \\
m_{A^{0}}^{2} & =\mu_{2}^{2}+\lambda_{S} v^{2} \\
m_{H^{ \pm}}^{2} & =\mu_{2}^{2}+\frac{1}{2} \lambda_{3} v^{2}
\end{aligned}
$$

where $\lambda_{L, S}$ are defined as:

$$
\lambda_{L, S}=\frac{1}{2}\left(\lambda_{3}+\lambda_{4} \pm \lambda_{5}\right) .
$$

This model involves 8 independent parameters: five $\lambda$, two $\mu_{i}$ and $v$. One parameter is eliminated by the minimization condition and the VEV is fixed by the $W$ boson mass. Finally, we are left with six independent parameters which we choose as follow:

$$
\left\{\mu_{2}^{2}, \lambda_{2}, m_{h}, m_{H^{ \pm}}, m_{H^{0}}, m_{A^{0}}\right\} .
$$

We note that the decoupling regime for the IDHM can be obtained by taking large $\mu_{2}^{2}$ while the quartic coupling $\lambda_{i}$ are taken within perturbativity limits, in such case the dark Higgses $m_{H^{0}, A^{0}, H^{ \pm}}$would be large while the $m_{h}$ is fixed by $\lambda_{1}$ parameter.

\subsection{Theoretical and experimental constraints}

In order to have vacuum stability, the parameters of the potential need to satisfy the positivity conditions. Namely, the potential should be bounded from below in all the directions of the field space, i.e. should not go to negative infinity for large field values. We have this set of constraints:

$$
\lambda_{1}>0, \quad \lambda_{2}>0, \quad \lambda_{3}+2 \sqrt{\lambda_{1} \lambda_{2}}>0 \quad \text { and } \quad \lambda_{3}+\lambda_{4}-\left|\lambda_{5}\right|>2 \sqrt{\lambda_{1} \lambda_{2}} .
$$


We ask that the perturbative unitarity is maintained in variety of scattering processes at high energy: scalar-scalar, scalar-vector, vector-vector. Using the equivalence theorem which replaces the $W$ and $Z$ bosons by the Goldstone bosons. We find a set of four matrices with entries are the quartic couplings, the diagonalization of these matrices gives us this set of eigenvalues [38]:

$$
\begin{aligned}
e_{1,2} & =\lambda_{3} \pm \lambda_{4}, \quad e_{3,4}=\lambda_{3} \pm \lambda_{5} \\
e_{5,6} & =\lambda_{3}+2 \lambda_{4} \pm 3 \lambda_{5} \\
e_{7,8} & =-\lambda_{1}-\lambda_{2} \pm \sqrt{\left(\lambda_{1}+\lambda_{2}\right)^{2}+\lambda_{4}^{2}} \\
e_{9,10} & =-3 \lambda_{1}-3 \lambda_{2} \pm \sqrt{9\left(\lambda_{1}-\lambda_{2}\right)^{2}+\left(2 \lambda_{3}+\lambda_{4}\right)^{2}} \\
e_{11,12} & =-\lambda_{1}-\lambda_{2} \pm \sqrt{\left(\lambda_{1}-\lambda_{2}\right)^{2}+\lambda_{5}^{2}} .
\end{aligned}
$$

By requiring that $e_{i} \leq 8 \pi$, we find the strongest constraint on $\lambda_{1,2}$ to be: $\lambda_{1,2} \leq \frac{4 \pi}{3}$. We also require that the parameters of the scalar potential remains perturbative, i.e.: $\left|\lambda_{i}\right| \leq 8 \pi$.

In order to have an inert vacuum the following constraint should be satisfied [39]:

$$
m_{h}^{2}, m_{H^{ \pm}}^{2}, m_{H^{0}}^{2}, m_{A^{0}}^{2}>0 \quad \text { and } \quad v^{2}>-\frac{\mu_{2}^{2}}{\sqrt{\lambda_{1} \lambda_{2}}} .
$$

The extra scalar particles affect quantum corrections to the $W$ and $Z$ bosons self energies. The corrections are parameterized by the oblique parameters, $S, T$ and $U$ [40] which are constrained from electroweak precision measurements. Taking the reference Higgs mass as $m_{h}=125 \mathrm{GeV}$ and $m_{\mathrm{t}}=173.5 \mathrm{GeV}$, the tolerated ranges are found at fixed $\mathrm{U}=0$ [41]:

$$
\Delta S=0.06 \pm 0.09, \quad \text { and } \quad \Delta T=0.10 \pm 0.07
$$

with correlated factor of +0.91 . Where $\Delta S=S^{\mathrm{IHDM}}-S^{\mathrm{SM}}$ and $\Delta T=T^{\mathrm{IHDM}}-T^{\mathrm{SM}}$. The formulas for $\Delta S$ and $\Delta T$ in the IHDM can be found in refs. [35, 42].

Searches of scalar particles ${ }^{1}$ of the IHDM at colliders [43] is not directly performed yet. However, several studies [44-46] applied SUSY searches involving two, three or multiple leptons with missing transverse energy $E_{T}^{\text {miss }}$ to the case of IHDM and set some limits on the dark Higges. We choose in our analysis: ${ }^{2}$

$$
m_{\Phi} \geq 100 \mathrm{GeV} \quad \text { where } \quad \Phi=H^{ \pm}, H^{0}, A^{0} .
$$

Finally, the magnitude of a possible Higgs boson signal at the LHC is characterized by the signal strength modifier, defined as $R_{\gamma \gamma}$ by:

$$
R_{\gamma \gamma}=\frac{\sigma(p p \rightarrow h \rightarrow \gamma \gamma)^{\mathrm{IHDM}}}{\sigma\left(p p \rightarrow h_{\mathrm{SM}} \rightarrow \gamma \gamma\right)}=\frac{B r(h \rightarrow \gamma \gamma)^{\mathrm{IHDM}}}{B r\left(h_{\mathrm{SM}} \rightarrow \gamma \gamma\right)}
$$

\footnotetext{
${ }^{1}$ These dark Higges have the same signature like neutralinos and charginos of the Minimal Supersymetric Standard Model (MSSM).

${ }^{2}$ If $H$ or $A$ is DM candidate then its mass could be as low as few $\mathrm{GeV}$.
} 
$h_{\mathrm{SM}}$ denotes a $125 \mathrm{GeV}$ SM Higgs boson. In our analysis below, while we will show points which satisfy theoretical and experimental constraints from our scans, we will highlight the points for which $R_{\gamma \gamma}$ is consistent with the measured $\mu_{\gamma \gamma}$ at the LHC. The latest publicly available measurements read $[47,48]$

$$
\begin{aligned}
\mu_{\gamma \gamma}^{\mathrm{CMS}} & =1.13 \pm 0.24 \\
\mu_{\gamma \gamma}^{\mathrm{ATLAS}} & =1.17 \pm 0.27 .
\end{aligned}
$$

\section{Radiative corrections to triple Higgs couplings $h h h$ and $h Z Z$}

In this section we calculate the one-loop radiative corrections to the trilinear Higgs couplings $h h h$ and $h Z Z$ in the SM and IHDM. The correction to those two couplings are part of the correction to double Higgs Strahlung process $e^{+} e^{-} \rightarrow Z h h$ which we will discuss in section 5 . Those couplings are given at the tree-level by:

$$
\begin{aligned}
\lambda_{h h h} & =\frac{-3 m_{h}^{2}}{v} \\
h Z_{\mu} Z_{\nu} & =\frac{e m_{W}}{s_{W} c_{W}^{2}} g_{\mu \nu} .
\end{aligned}
$$

As one can see, both couplings $h h h$ and $h Z Z$ involve only SM parameters. Those couplings receive corrections from one-loop diagrams. The one-loop effects from the SM particles have been studied in [50-54] for $h h h$ and in [49] for $h Z Z$. These effects are dominated by the top quark loops which does not exceed $10 \%$ for $h h h$ and $1.5 \%$ for $h Z Z$.

New physics effects to hhh coupling have been analyzed in the context of the Two Higgs Doublet Model [50, 51] and the MSSM [53, 54]. It was found that these effects can enhance significantly this coupling in a wide range of parameter space. Furthermore, these corrections depend on the model and hence any deviation from the SM tree level relation (3.1) by more than $10 \%$ would be an evidence for the presence of new physics.

The coupling $h Z Z$ have been analyzed in the framework of the two Higgs doublet model [52] and it has been found that the effect is rather small $1 \%$ to $2 \%$.

We have calculated the radiative corrections to the tree level triple Higgs coupling $h h h$ and $h Z Z$ both in the SM and IHDM in the Feynman gauge including all the particles of the model in the loops. The Feynman diagrams from IHDM contributing to $\lambda_{h h h}$ coupling are shown in figure 1. The one-loop amplitudes are calculated using dimensional regularization. The calculation was done with the help of FeynArts and FormCalc [55-58] packages. Numerical evaluation of the one-loop scalar integrals have been done with LoopTools [59-61]. We have checked both numerically and analytically the UV finiteness of the amplitudes.

In order to do that, we have considered $h h h$ and $h Z Z$ at one-loop level:

i) for $h h h$, we considered the decay of an off-shell Higgs boson into a Higgs boson pairs $h^{*}(q) \rightarrow h\left(k_{1}\right) h\left(k_{2}\right)$ at the one-loop level. Where $q, k_{1}$ and $k_{2}$ are the 4-momenta of the three particles satisfying on shell conditions $k_{1}^{2}=k_{2}^{2}=m_{h}^{2}$ for final state Higgs pairs and an off shell condition $q^{2} \neq m_{h}^{2}$ for the decaying Higgs. 


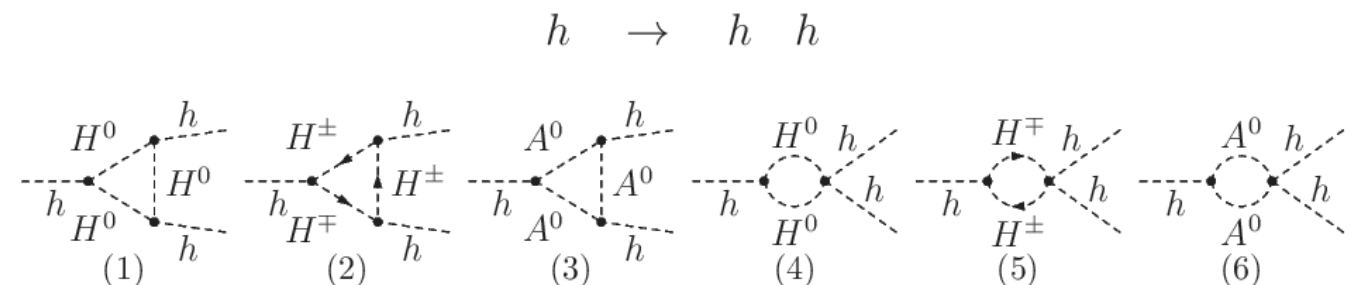

(1)

(2)

(3)

(4)

(5)

(6)

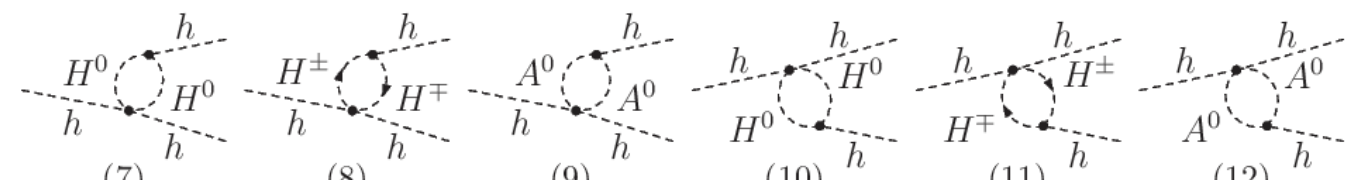

(7)

(8)

(9)

(10)

(11)

(12)

Figure 1. One loop Feynman diagrams contributing to $h h h$ in the IHDM. S stands for $H^{ \pm}, G^{ \pm}, G^{0}$ and $\mathrm{V}=W^{ \pm}$.

ii) For $h Z Z$, we follow ref [52] and write:

$$
M_{h Z Z}^{\mu \nu}\left(q^{2}=m_{h}^{2}, k_{1}^{2}, k_{2}^{2}\right)=M_{1}^{h Z Z} g^{\mu \nu}+M_{2}^{h Z Z} \frac{k_{1}^{\nu} k_{2}^{\mu}}{m_{Z}^{2}}+M_{3}^{h Z Z} i \epsilon^{\mu \nu \rho \sigma} \frac{k_{1 \rho} k_{2 \sigma}}{m_{Z}^{2}},
$$

where $k_{1}$ and $k_{2}$ are the momenta of outgoing $Z$ bosons. We assume that the decaying Higgs and one of the $\mathrm{Z}$ boson are on-shell $q^{2}=m_{h}^{2}, k_{1}^{2}=m_{Z}^{2}$ while the other $\mathrm{Z}$ boson is off-shell $k_{2}^{2}=\left(m_{h}-m_{Z}\right)^{2}$. Using power counting arguments, it is expected that $M_{1}^{h Z Z}$ receives the highest power contribution of the heavy fermions masses. Therefore, in what follow we will take into account only the $M_{1}^{h Z Z}$ form-factor to $h Z Z$ coupling.

Since we are dealing with a processes at the one-loop level, a systematic treatment of the UV divergences have to be considered. We will use the on-shell renormalization scheme in which the input parameters coincide with the physical masses and couplings [62, 63]. In the on shell scheme, a redefinition of the fields and parameters is performed. This redefinition cast the Lagrangian into a bare Lagrangian and counter-term. The counterterms are calculated by specific renormalization conditions which allow us to cancel the UV divergences of the diagrams with loops. Furthermore, since we have three Higgs as external particles and there is no mixing between the SM doublet $H_{1}$ and the inert doublet $H_{2}$, we do not need to renormalize the particle content of the scalar potential of the IHDM. The tree level coupling hhh eq. (3.1) depends only on Higgs mass and the vev as in the SM, then the renormalization procedure will be the same as in the SM [62,63]. We redefine the SM fields and parameters as follow:

$$
\begin{aligned}
m_{V}^{2} & \rightarrow m_{V}^{2}+\delta m_{V}^{2}, \quad V=W^{ \pm}, Z \\
m_{h}^{2} & \rightarrow m_{h}^{2}+\delta m_{h}^{2} \\
s_{W} & \rightarrow s_{W}+\delta s_{W} \\
e & \rightarrow\left(1+\delta Z_{e}\right) e
\end{aligned}
$$




$$
\begin{aligned}
t & \rightarrow t+\delta t \\
W^{\mu} & \rightarrow Z_{W}^{1 / 2} W^{\mu}=\left(1+\frac{1}{2} \delta Z_{W}\right) W^{\mu} \\
Z^{\mu} & \rightarrow\left(1+\frac{1}{2} \delta Z_{Z Z}\right) Z^{\mu}+\frac{1}{2} \delta Z_{Z A} A^{\mu} \\
A^{\mu} & \rightarrow\left(1+\frac{1}{2} \delta Z_{A A}\right) A^{\mu}+\frac{1}{2} \delta Z_{A Z} Z^{\mu} \\
h & \rightarrow Z_{h}^{1 / 2} h=\left(1+\frac{1}{2} \delta Z_{h}\right) h
\end{aligned}
$$

where $s_{W}=\sin \theta_{W}$ is the Weinberg angle and $t=v\left(\mu_{1}^{2}-\lambda_{1} v^{2}\right)$ is the tadpole which is zero at tree level once the minimization condition is used but will receives again finite radiative corrections at the one-loop level. To ensure that the VEV is the same in all orders of perturbation theory, it is well known that one need to renormalize the Higgs tadpole: i.e., all Higgs tadpole amplitudes $T$ are absorbed into the counter-term $\delta t$. Thus, we put the first condition:

$$
\hat{T}=\delta t+T=0 .
$$

The mass counter-terms are fixed by the on shell conditions $[62,63]$ :

$$
\begin{aligned}
\operatorname{Re} \hat{\Sigma}_{T}^{V V}\left(m_{V}^{2}\right) & =0, \quad V=W, Z \\
\operatorname{Re} \hat{\Sigma}_{h}\left(m_{h}^{2}\right) & =0 .
\end{aligned}
$$

The field renormalization constants are fixed by imposing that the residue of the two point Green functions to be equal to unity and the mixing $\gamma$-Z vanishes for $k^{2}=m_{Z}^{2}$. While the electric charge renormalization constant $\delta Z_{e}$ is treated like in quantum electrodynamics and is fixed from the $e^{+} e^{-} \gamma$ vertex. The renormalized three point function $\hat{\Gamma}_{e^{+} e^{-\gamma}}^{\mu}$ satisfies at the Thomson limit:

$$
\hat{\Gamma}_{e^{+} e^{-\gamma}}^{\mu}\left(\not p_{1}=\not p_{2}=m, q^{2}=0\right)=e .
$$

Furthermore, the counter-term $\delta s_{W}$ can be obtained from the on-shell definition $s_{W}^{2}=$ $1-\frac{m_{W}^{2}}{m_{Z}^{2}}$ as a function of $\delta m_{W}$ and $\delta m_{Z}$.

Inserting these redefinitions into the Lagrangian, we find the following counter term for $h h h$ and $h Z Z[62,63]$ :

$$
\begin{aligned}
\delta \mathcal{L}_{h h h} & =\frac{-3 e^{2}}{2 s_{W}} \frac{m_{h}^{2}}{m_{W}}\left(\delta Z_{e}-\frac{\delta s_{W}}{s_{W}}+\frac{\delta m_{h}^{2}}{m_{h}^{2}}+\frac{e}{2 s_{W}} \frac{\delta t}{M_{W} m_{h}^{2}}-\frac{\delta m_{W}^{2}}{2 m_{W}^{2}}+\frac{3}{2} \delta Z_{h}\right) h^{3} \\
\delta \mathcal{L}_{h Z Z} & =\delta M_{1}=\frac{e m_{W}}{s_{W} c_{W}^{2}}\left(\delta Z_{e}+\frac{2 s_{W}^{2}-c_{W}^{2}}{c_{W}^{2} s_{W}} \delta s_{W}+\frac{\delta m_{W}^{2}}{2 m_{W}^{2}}+\frac{1}{2} \delta Z_{H}+\delta Z_{Z}\right) h Z^{\mu} Z^{\nu} .
\end{aligned}
$$

By adding the un-renormalized amplitude for $h h h$ and $h Z Z$ to the above corresponding counter-terms, one finds the renormalized amplitudes

$$
\begin{aligned}
\hat{\Gamma}_{h h h}\left(q^{2}, m_{h}^{2}, m_{h}^{2}\right) & =\Gamma_{h h h}^{1 \text {-loop }}\left(q^{2}, m_{h}^{2}, m_{h}^{2}\right)+\delta \mathcal{L}_{h h h} \\
\hat{\Gamma}_{h Z Z}\left(m_{h}^{2}, m_{Z}^{2},\left(m_{h}-m_{Z}\right)^{2}\right) & =M_{1 h Z Z}^{\text {tree }}+M_{1}^{1-\text { loop }}+\delta \mathcal{L}_{h Z Z}
\end{aligned}
$$


which becomes UV finite. For our numerical illustrations, we define the following ratios:

$$
\begin{aligned}
\Delta \Gamma_{h h h} & =\frac{\operatorname{Re}\left(\tilde{\Gamma}_{h h h}\left(q^{2}\right)\right)}{\lambda_{h h h}} \\
\Delta \Gamma_{h Z Z} & =\frac{\hat{\Gamma}_{h Z Z}^{I D M}-\hat{\Gamma}_{h Z Z}^{\mathrm{SM}}}{M_{1 h Z Z}^{\mathrm{tree}}}=\frac{M_{1}^{\mathrm{IHDM}}-M_{1}^{\mathrm{SM}-\text { tree }}}{M_{1}^{\mathrm{SM}-\text { tree }}} .
\end{aligned}
$$

Where $\hat{\Gamma}_{h h h}$ is the renormalized vertex.

\section{Numerical results}

\subsection{SM case}

In our numerical analysis, the parameters are chosen as follow:

$$
m_{\mathrm{t}}=173.5 \mathrm{GeV}, \quad m_{W}=80.3996 \mathrm{GeV}, \quad m_{Z}=91.1875 \mathrm{GeV}, \quad \alpha=\frac{1}{127.934}
$$

and the on-shell definition of the Weinberg angle: $\sin ^{2} \theta_{W}=1-\frac{m_{W}^{2}}{m_{Z}^{2}}$.

In the SM, the dominant contribution to $\Delta \Gamma_{h h h}(\mathrm{SM})$ comes from top quark loops [50, $51,53,54]$. We have computed the top contribution and shown that it is in perfect agreement with refs. $[50,51]$. We have also isolated and evaluated the other SM contributions without fermions. It turns out that this bosonic contribution is of the order of $5 \%$ for large $q$.

In figure 2 (left), the fermionic and bosonic contributions are shown as a function of $q$. In the limit where $q$ is small, the correction due to the quark loop is negative. However for $2 m_{h}<q<2 m_{\mathrm{t}}$ the total correction change sign due to the loop effect and reaches its maximum at $q=2 m_{\mathrm{t}}$. Far from threshold, $\Delta \Gamma_{h h h}(\mathrm{SM})$ is dominated by top-quark contributions.

In figure 2 (right), we show the radiative corrections to $h Z Z$ in the SM. We present separately the fermionic corrections which are dominated by the top contributions and the bosonic contributions. The total corrections to $h Z Z$ is of the order of $2 \%$. In this plot, we also shift the triple Higgs SM coupling $\lambda_{h h h}^{\mathrm{SM}}$ by $\lambda_{h h h}^{\mathrm{SM}}(1+\Delta)$, where $\Delta$ represents any deviation from SM coupling. As one can see from the green line, the sensitivity to $\Delta$ is rather mild. Due to custodial symmetry, it is expected that $h W W$ coupling will enjoy similar effect as $h Z Z$ and that is why we illustrate only the case of $h Z Z$.

\subsection{IHDM case}

Here, we will show our numerical analysis for the triple coupling of the Higgs in the IHDM taking into account: unitarity, perturbativity, false vacuum as well as vacuum stability constraints described above. We take the mass of the SM Higgs $m_{h}=125 \mathrm{GeV}$ and the masses of the inert particles to be degenerate, i.e.: $m_{H^{ \pm}}=m_{H^{0}}=m_{A^{0}}=m_{\Phi}$. For the 

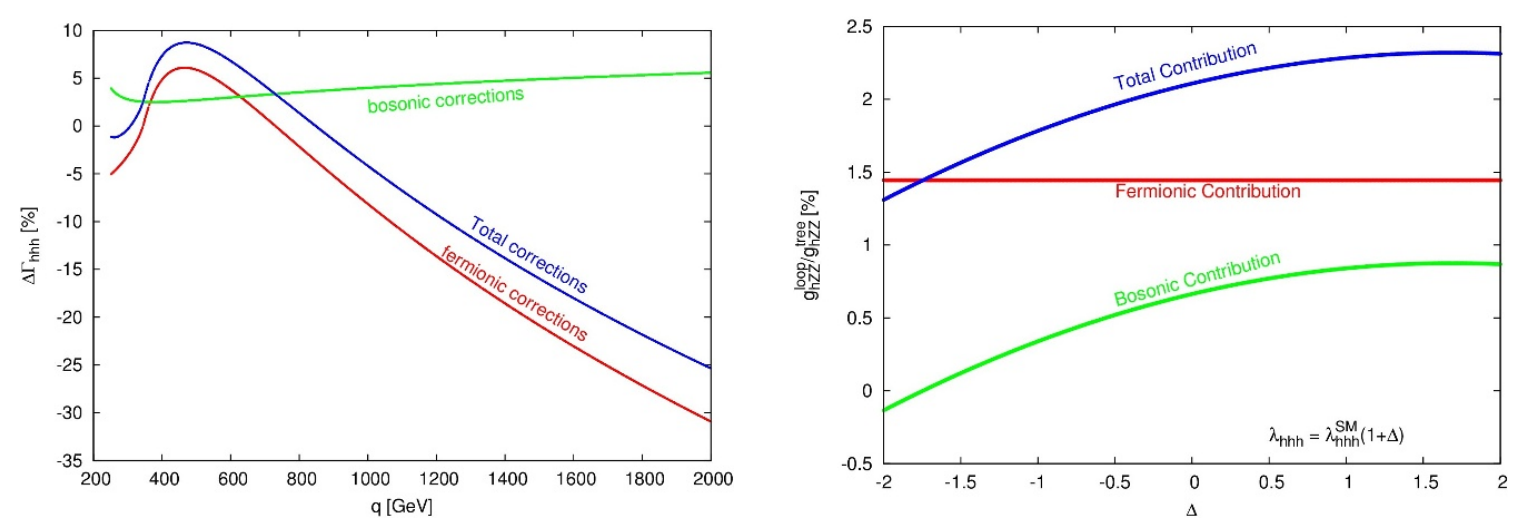

Figure 2. (Left) $\Delta \Gamma_{h h h}(\mathrm{SM})$ as a function of $h^{*}$ momentum $q$. (Right) $\Delta \Gamma_{h Z Z}(\mathrm{SM})$ as a function of $\Delta$ which is the size of deviation from SM triple coupling $\lambda_{h h h}=\lambda_{h h h}^{\mathrm{SM}}(1+\Delta)$. It is shown: the fermionic contribution, the bosonic one as well as the total contribution.

other parameters, we perform the following scan:

$$
\begin{aligned}
100 \mathrm{GeV} & \leq m_{\Phi} \leq 500 \mathrm{GeV} \\
-25 \times 10^{5}(\mathrm{GeV})^{2} & \leq \mu_{2}^{2} \leq 9 \times 10^{5}(\mathrm{GeV})^{2} \\
0 & <\lambda_{2} \leq \frac{4 \pi}{3}
\end{aligned}
$$

We plot in figure 3 (left) the relative corrections to the triple coupling as a function of $\lambda_{2}$. The theoretical constraints put a limit on $\lambda_{2}$ parameter which is $\lambda_{2} \leq \frac{4 \pi}{3}$. One can see from figure 3 that the corrections are maximized for $\lambda_{2} \leq 2$ and decrease for $\lambda_{2}>2$. In our following analysis, We will take $\lambda_{2}=2$ in order to maximize the effect from $\lambda_{2}$.

In figure 3 (right), we plot the relative corrections to the triple coupling $h h h$ in the plane $\left(m_{\Phi}, \mu_{2}^{2}\right)$ for a fixed $q=300 \mathrm{GeV}$ and $\lambda_{2}=2$. One can see that the corrections are very important in a large part of the parameter space with an enhancement up to $280 \%$ for large values of $m_{\Phi}$ and negative $\mu_{2}^{2}$. Furthermore, these corrections are increasing, for a fixed value of $m_{\Phi}$, while $\mu_{2}^{2}$ is decreasing. The maximum of the corrections is reached for $\mu_{2}^{2} \approx-30000(\mathrm{GeV})^{2}$.

Moderate or very small corrections which can be in the range $[-50,50] \%$ are also possible for large area of parameter space with low $m_{\Phi} \leq 300 \mathrm{GeV}$ and any positive $\mu_{2}^{2}$. It is also important to note that LHC constraint from diphoton at the $2 \sigma$ level exclude light charged Higgs $100<m_{H^{ \pm}}<175 \mathrm{GeV}$ and negative $\mu_{2}^{2}$ : left-down corner of the scatter plot. In figure 4 we show the relative corrections $\Delta \Gamma_{h h h}($ IHDM) as a function of the momentum of the off-shell decaying Higgs $q$ and for $m_{\Phi}$ and $\mu_{2}^{2}$ as shown in eq. (4.1). It is clear that for low $100<m_{\Phi}<200 \mathrm{GeV}$, the corrections are small like in the SM case except at the threshold regions where we have $\Delta \Gamma_{h h h} \sim-40 \%$ due to the opening of $h^{*} \rightarrow \Phi \Phi$. For large $m_{\Phi}$ these corrections could be extremely large exceeding $100 \%$ in large area of parameter space. The corrections are amplified by the opening of the threshold channel $h^{*} \rightarrow \Phi \Phi$. This is visible on the left panel of figure 4 where we can see a kink for $q=1000 \mathrm{GeV}$ which correspond to threshold effect $h^{*} \rightarrow \Phi \Phi$ with $m_{\Phi} \approx 500 \mathrm{GeV}$. As it is shown, negative 

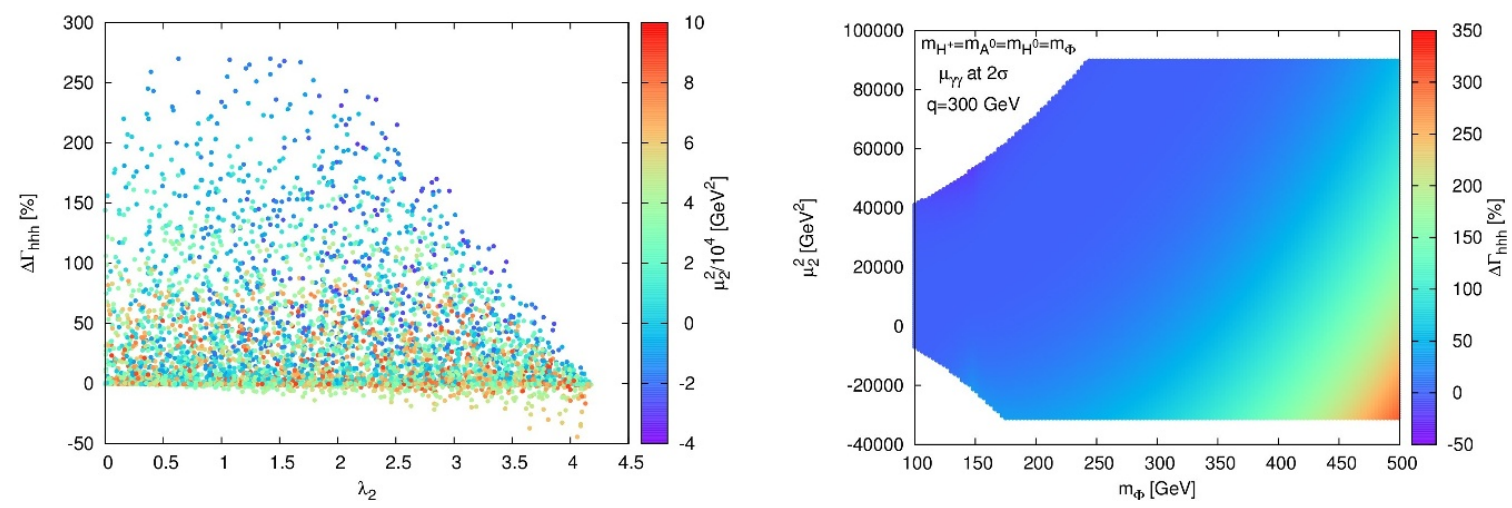

Figure 3. Left: $\Delta \Gamma_{h h h}(\mathrm{IHDM})$ as a function of $\lambda_{2}$. Right: scatter plot for $\Delta \Gamma_{h h h}$ in the plan $\left(m_{\Phi}, \mu_{2}^{2}\right)$ for $q=300 \mathrm{GeV}$. Right column shows the size of the corrections. $m_{\Phi}$ and $\mu_{2}^{2}$ are scanned as in eq. (4.1).

values for $\mu_{2}^{2}$ give large corrections to the triple Higgs coupling. This is because in our assumption of taking degenerate Higges $m_{H^{0}}=m_{A^{0}}=m_{H^{ \pm}}=m_{\Phi}$ one can show that $\lambda_{4}=\lambda_{5}=0, \lambda_{3}=\frac{2}{v^{2}}\left(m_{H^{ \pm}}^{2}-\mu_{2}^{2}\right)$ and therefore the triple couplings are given by

$$
\begin{aligned}
h H H & =\lambda_{L} v=\frac{2}{v}\left(m_{\Phi}^{2}-\mu_{2}^{2}\right) \\
h A A & =\lambda_{A} v=\frac{2}{v}\left(m_{\Phi}^{2}-\mu_{2}^{2}\right) \\
h H^{+} H^{-} & =\lambda_{3} v=\frac{2}{v}\left(m_{\Phi}^{2}-\mu_{2}^{2}\right) .
\end{aligned}
$$

It is clear that those couplings get stronger for negative $\mu_{2}^{2}$. One need to keep in mind that even for such a large $\mu_{2}^{2}$, all the $\lambda_{i}$ involved are still within unitarity range. Similar effects have obtained in the framework of two Higgs doublet Model [50, 51]. We now examine the effect of the radiative corrections on the triple coupling in the case where the invisible decay $h \rightarrow H H$ is open. This is illustrated in figure 5 (left) and right. In figure 5 (left) we impose both $\left|\lambda_{L}\right|<0.02$ required by dark matter constraints as well as best fit limit on the invisible branching ratio $\mathrm{Br}(h \rightarrow$ invisible $) \leq 10 \%$ (left) and $\mathrm{Br}(h \rightarrow$ invisible $) \leq 20 \%$ (right) [6469]. It is clear from left panel that with dark matter constraint, the size of the corrections and the range of $\mu_{2}^{2}$ are smaller than in the previous case where the invisible decay was closed. The large corrections observed for high $m_{\Phi}$ are mainly due to the charged Higgs loops.

In order to exhibit the decoupling behavior on the triple Higgs coupling, we illustrate in figure $6 \Delta \Gamma_{h h h}(\mathrm{IHDM})$ as a function of $m_{\Phi}$. To have large dark Higgs masses $m_{\Phi}$ we take $\mu_{2}^{2}$ to be large enough: $\left[-10^{6}, 10^{7}\right] \mathrm{GeV}^{2}$. When we increase $\mu_{2}^{2}$ in the range $\left[-10^{6}, 10^{7}\right] \mathrm{GeV}^{2}$ we have $m_{\Phi} \in[0.1,3] \mathrm{TeV}$. We see that the decoupling effect occurs when the $m_{\Phi}$ is larger than $1.5 \mathrm{TeV}$. It is clear from the plot that $\Delta \Gamma_{h h h}$ reaches its maximum for $m_{\Phi} \approx 500 \mathrm{GeV}$ and decreases to SM value for large $m_{\Phi}$.

In figure 7 we illustrate the IHDM effect on $h Z Z$ coupling. Similar to the triple Higgs coupling, we fix $\lambda_{2}=2$ and scan over $\mu_{2}^{2}$ and $m_{\Phi}=m_{H}=m_{A}=m_{H \pm}$ as in eq. (4.1). In 

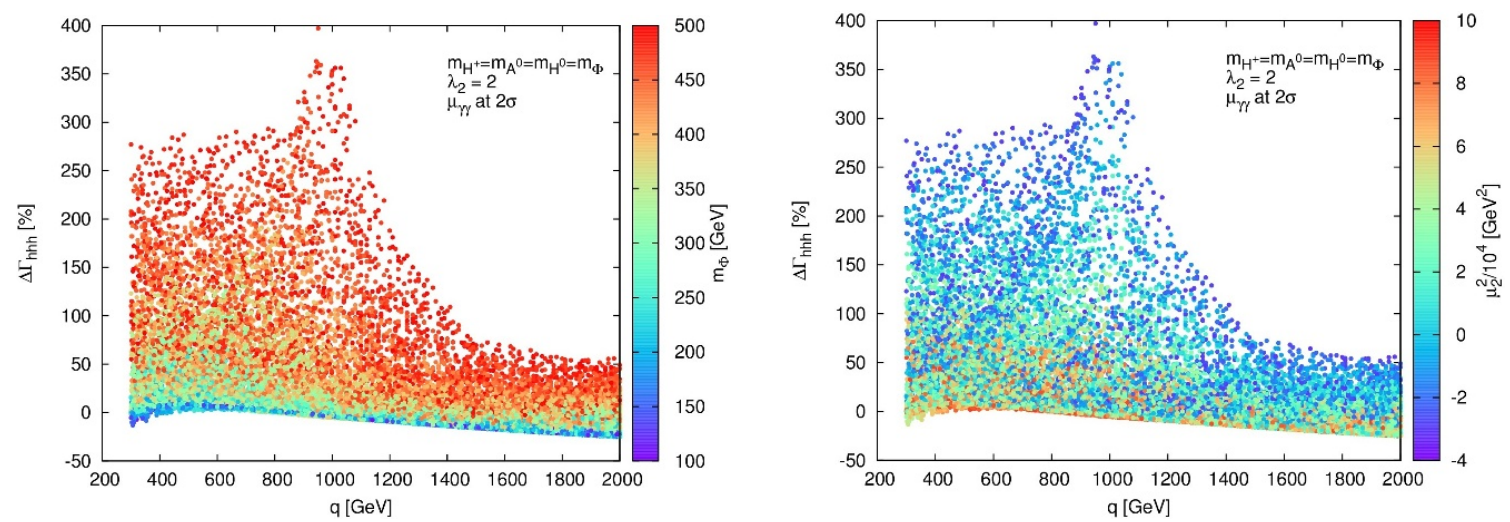

Figure 4. $\Delta \Gamma_{h h h}(\mathrm{IHDM})$ as a function of $q$ and where $m_{\Phi}$ and $\mu_{2}^{2}$ are scanned as in eq. (4.1). The right column shows the values of $m_{\Phi}$ (left panel) and $\mu_{2}^{2}$ (right panel).
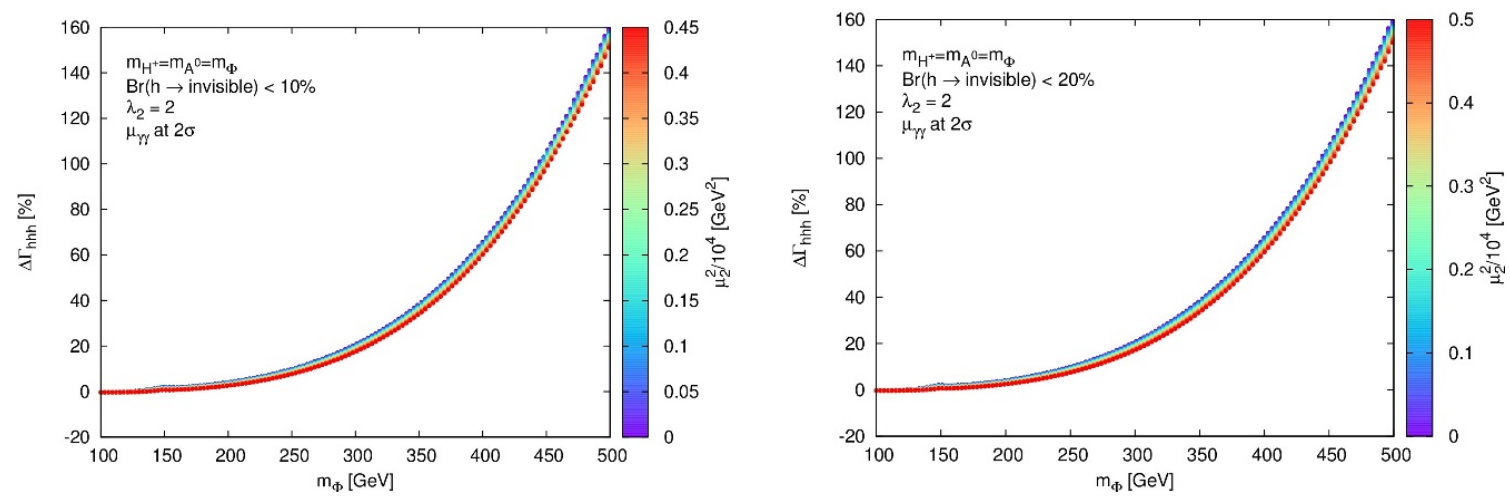

Figure 5. $\Delta \Gamma_{h h h}$ as a function of $m_{\Phi}$ for $q=300 \mathrm{GeV}$ with $\mu_{2}^{2}$ in the range given by the eq. (4.1) and invisible decay of the Higgs $(h \rightarrow H H)$ is open. Left panel with dark matter constraint which restricts $\left|\lambda_{L}\right|$ to be in the range $\left|\lambda_{L}\right|<0.02$. Right panel without dark matter constraint.
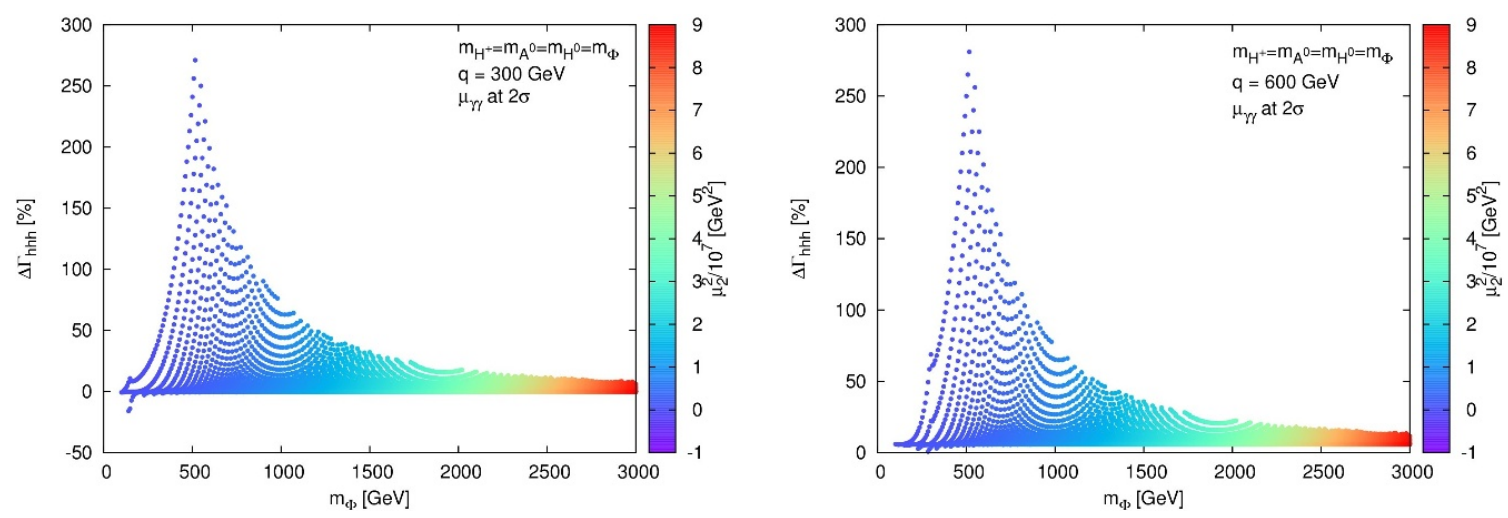

Figure 6. Left: $\Delta \Gamma_{h h h}(\mathrm{IHDM})$ as a function of $m_{\Phi}$ for fixed $\lambda_{2}=2$ and $q=300 \mathrm{GeV}$ (left), while $q=600 \mathrm{GeV}$ in the right. Right column shows the range of $\mu_{2}^{2}$.

figure 7 (left) we show scatter plot for $\Delta \Gamma_{h Z Z}$ in the plan $\left(\mu_{2}^{2}, m_{\Phi}\right)$, contrarily to the triple coupling $h h h$ the effects are rather small of the same size as in the SM case. In figure 7 

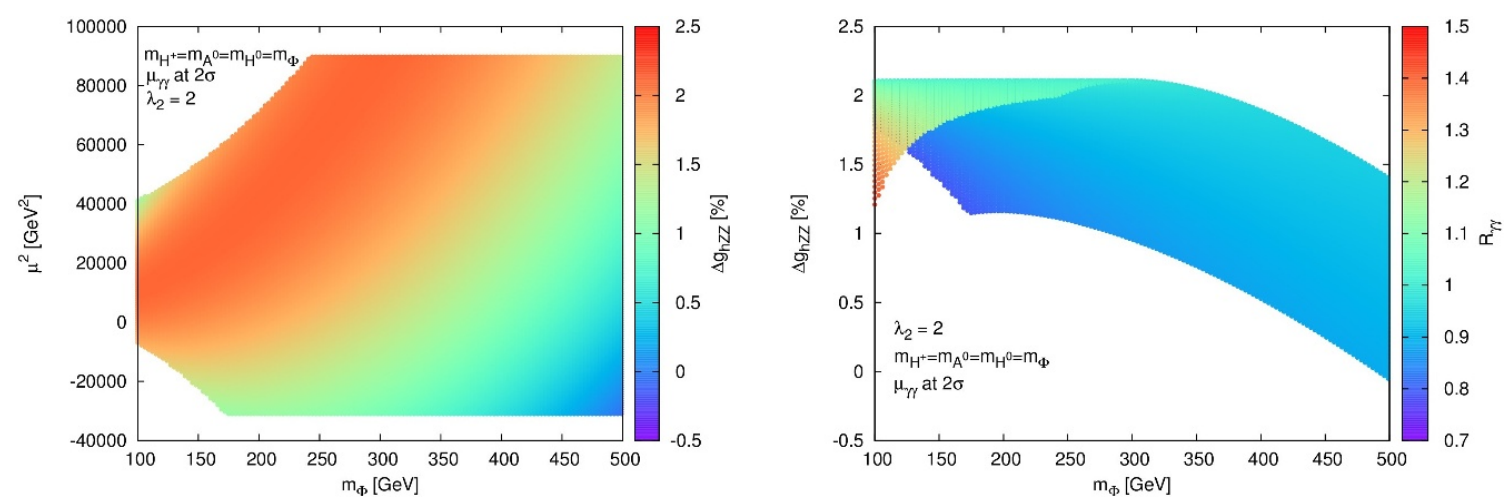

Figure 7. Left: scatter plot for $\Delta \Gamma_{h Z Z}$ in the plan $\left(m_{\Phi}, \mu_{2}^{2}\right)$ with $\lambda_{2}=2$, left column represent the size of the corrections. Right: $\Delta \Gamma_{h Z Z}$ as function of $m_{\Phi}$ with $\mu_{2}^{2}$ in the same range as in the left panel. Right column shows the size of $R_{\gamma \gamma}$.

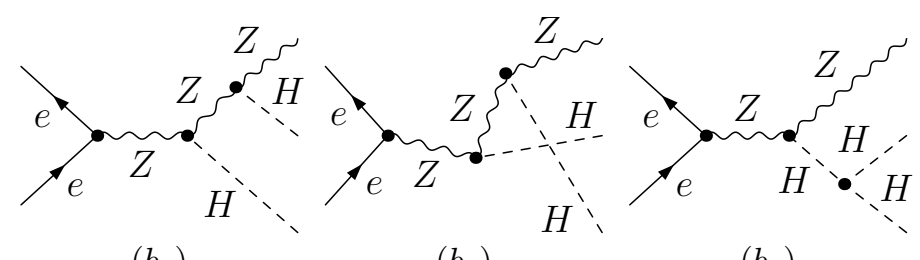

$\left(b_{1}\right)$

$\left(b_{2}\right)$

$\left(b_{3}\right)$

Figure 8. Feynman diagrams contributing to $e^{+} e^{-} \rightarrow Z h h$ at the tree level in SM.

(right) we show the corrections to $h Z Z$ coupling and also the value of the corresponding $R_{\gamma \gamma}$ within $2 \sigma$ range.

\section{Radiative corrections to $e^{+} e^{-} \rightarrow h h Z$ in the IHDM}

\section{$5.1 e^{+} e^{-} \rightarrow h h Z$ in $\mathrm{SM}$}

At $e^{+} e^{-}$LC, the triple Higgs coupling can be probed by double Higgs-Strahlung process $e^{+} e^{-} \rightarrow h h Z$ depicted in figure 8 and WW fusion process $e^{+} e^{-} \rightarrow W W^{*} \nu \bar{\nu} \rightarrow h h \nu \bar{\nu}$. Note that $e^{+} e^{-} \rightarrow h h Z$ process arise in $s$-channel only and hence its cross section can be probed more efficiently at low energies above the threshold (typically between $350 \mathrm{GeV}$ and $500 \mathrm{GeV}$ ). While, at high energies, for $\sqrt{s} \gtrsim 700 \mathrm{GeV}$, the trilinear Higgs-coupling is better probed through the process $e^{+} e^{-} \rightarrow \nu \bar{\nu} h h$ assuming the SM. In figure 8 we show the Feynman diagrams contributing to $e^{+} e^{-} \rightarrow h h Z$. When extracting the triple Higgs coupling $h h h$ from this process only diagram figure $8\left(b_{1}\right)$ is concerned, the other diagrams $b_{2,3}$ are considered as a background.

We illustrate in figure 9 the tree level cross section for $e^{+} e^{-} \rightarrow h h Z$ as a function of $\Delta$ for center of mass energy $500 \mathrm{GeV}$ and $800 \mathrm{GeV}$, where $\Delta$ is the shift of the SM triple Higgs coupling $\lambda_{h h h}=\lambda_{h h h}^{\mathrm{SM}}(1+\Delta)$. It is clear that for $\Delta>0$ the cross section is enhanced while for $\Delta<0$ the cross section is reduced with respect to $\mathrm{SM}$ value.

At $e^{+} e^{-}$LC, the triple Higgs coupling can be probed by double Higgs-Strahlung process $e^{+} e^{-} \rightarrow h h Z$ depicted in figure 8 and WW fusion process $e^{+} e^{-} \rightarrow W W^{*} \nu \bar{\nu} \rightarrow h h \nu \bar{\nu}$. Note 


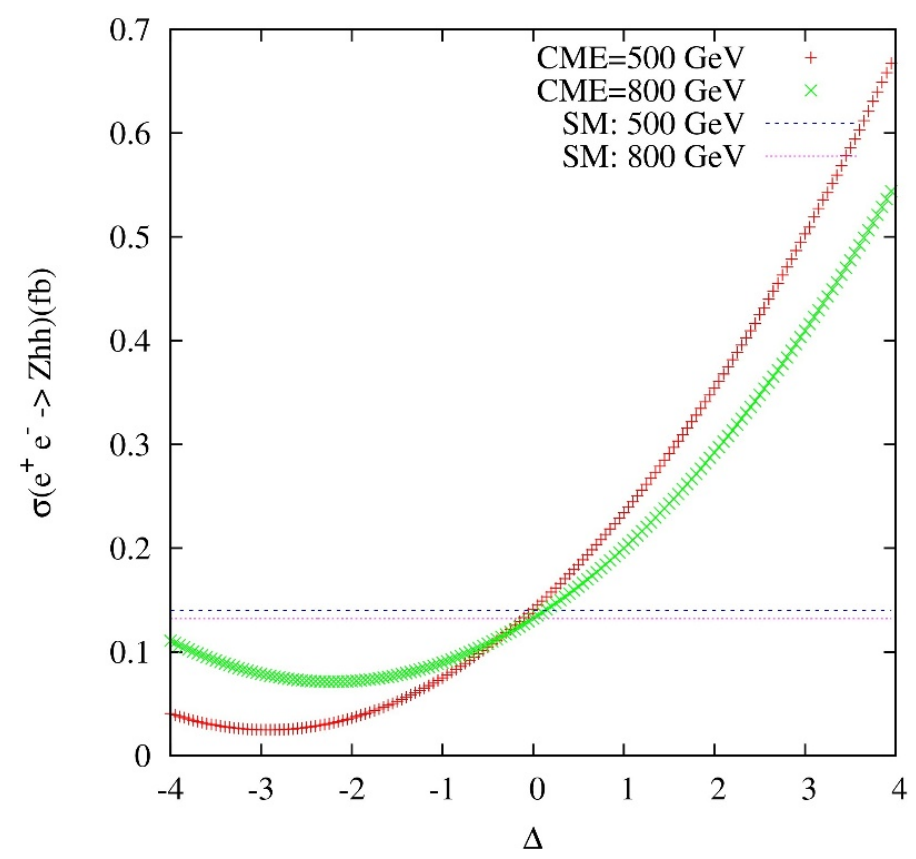

Figure 9. Cross section of $e^{+} e^{-} \rightarrow Z h h$ as a function of $\Delta$ where $\lambda_{h h h}=\lambda_{h h h}^{\mathrm{SM}}(1+\Delta)$ for $\sqrt{s}=500,800 \mathrm{GeV}$.

that $e^{+} e^{-} \rightarrow h h Z$ process arise in $s$-channel only and hence its cross section can be probed more efficiently at low energies above the threshold (typically between $350 \mathrm{GeV}$ and $500 \mathrm{GeV}$ ). While, at high energies, for $\sqrt{s} \gtrsim 700 \mathrm{GeV}$, the trilinear Higgs-coupling is better probed through the process $e^{+} e^{-} \rightarrow \nu \bar{\nu} h h$ assuming the SM. In figure 8 we show the Feynman diagrams contributing to $e^{+} e^{-} \rightarrow h h Z$. When extracting the triple Higgs coupling $h h h$ from this process only diagram figure $8\left(b_{1}\right)$ is concerned, the other diagrams $b_{2,3}$ are considered as a background.

We illustrate in figure 9 the tree level cross section for $e^{+} e^{-} \rightarrow h h Z$ as a function of $\Delta$ for center of mass energy $500 \mathrm{GeV}$ and $800 \mathrm{GeV}$, where $\Delta$ is the shift of the SM triple Higgs coupling $\lambda_{h h h}=\lambda_{h h h}^{\mathrm{SM}}(1+\Delta)$. It is clear that for $\Delta>0$ the cross section is enhanced while for $\Delta<0$ the cross section is reduced with respect to SM value.

\subsection{One-loop corrections to $e^{+} e^{-} \rightarrow Z h h$}

We study in this section the effects of the one-loop radiative corrections to the Higgs trilinear self-coupling calculated in the previous section on the double Higgs-Strahlung process via $Z$ boson exchange. In the context of the SM, the $\mathcal{O}(\alpha)$ electroweak corrections have been studied in [70] and it was found that these corrections are of the order $10 \%$. However, these loop effects can be very large in beyond SM enhancing the total cross section by about 2 orders of magnitude in particular in models with extended Higgs sector. As outlined above, at the tree level, $e^{+} e^{-} \rightarrow h h Z$ have three diagrams as depicted in figure 8 . Only the first diagram contributes to the signal while the others are considered as a background. In our analysis, we include one-loop correction only to the triple Higgs coupling $h h h$ which is expected to give sizeable contribution. Only this correction contributes to 
the signal. Therefore, we did not include corrections to the initial state vertex $e^{+} e^{-} Z$, to the self energies of $Z-Z$ and $\gamma-Z$ mixing, to the $h Z Z$ coupling and also we did not include the initial state radiation. In fact these corrections are well known in the SM and are not expected to deviate that much in the IHDM as we already show in the previous section for $h Z Z$ coupling. Moreover, we will not include corrections to $e^{+} e^{-} \rightarrow h h Z$ coming from boxes and pentagon diagrams. The one-loop amplitude can be written as follow:

$$
\mathcal{M}=\mathcal{M}_{\text {tree }}+\mathcal{M}_{\text {loop }}
$$

The squared amplitude at the one-loop level is then:

$$
|\mathcal{M}|^{2}=\left|\mathcal{M}_{\text {tree }}\right|^{2}+2 \operatorname{Re}\left\{\mathcal{M}_{\text {tree }}^{*} \mathcal{M}_{\text {loop }}\right\}+\mathcal{O}\left(\alpha^{2}\right) .
$$

Thus, the cross section is written as:

$$
\sigma=\frac{1}{(2 \pi)^{2}} \int \prod_{k=1}^{3} \frac{d^{3} \mathbf{p}_{k}}{2 E_{k}} \delta^{(4)}\left(q_{1}+q_{2}-p_{1}-p_{2}-p_{3}\right) \sum_{\text {spin, pola. }}|\mathcal{M}|^{2}
$$

where $q_{1}$ and $q_{2}$ are the four momenta of the incoming electron and positron, $p_{1}, p_{2}$ and $p_{3}$ are the four momenta of the outgoing particles, and the factor $\frac{1}{(2 \pi)^{2}}$ arises from the flux of the initial particles.

For our studies, we define the ratio $\Delta \sigma$ by:

$$
\Delta \sigma=\frac{\sigma_{\text {total }}-\sigma_{\text {tree }}}{\sigma_{\text {tree }}}=\frac{\sigma_{\text {loop }}}{\sigma_{\text {tree }}} .
$$

Where $\sigma_{\text {total }}=\sigma_{\text {tree }}+\sigma_{\text {loop }}$. This ratio measures the relative correction of the IHDM to the cross section with respect to the tree level result.

As stated before, in our analysis we will take into account the theoretical and experimental constraints discussed in the second section assuming the parameters to rely in the range given by eq. (4.1). The phase space and evaluation of the one-loop squared amplitude has been performed with FormCalc [55-58] with the help of LoopTools to evaluate numerically the one-loop scalar integrals. We have used the same on-shell renormalization scheme explained in the previous section.

In figure 10, we have plotted the ratio $\Delta \sigma$ versus $m_{\Phi}$ for center of mass energy $\sqrt{s}=$ $500 \mathrm{GeV}$. We assume again that: $\lambda_{2}=2$, the dark Higges to be degenerate $m_{H}=m_{A}=$ $m_{H \pm}=m_{\Phi}$ and perform a scan over $\mu_{2}^{2}$ and $m_{\Phi}$. From this plot one can see that the corrections can reach $160 \%$ for high dark Higgs masses $m_{\Phi} \approx 500 \mathrm{GeV}$ and are negative for low dark Higgs masses $100 \mathrm{GeV} \leq m_{\Phi} \leq 270 \mathrm{GeV}$ depending on the value of $\mu_{2}^{2}$. One can see that the suppression of the total cross section can reach $-15 \%$ for $m_{\Phi}=140 \mathrm{GeV}$ while the enhancement is predominant in most part of the parameter space.

To understand this, let us remind first that in large area of parameter space the correction to the triple Higgs coupling parameter $\Delta \Gamma_{h h h}$ is positive (see section 4 figure 3). Moreover, according to the plot figure 9, if this correction is positive this lead to an enhancement of the total cross section and vice-versa. 

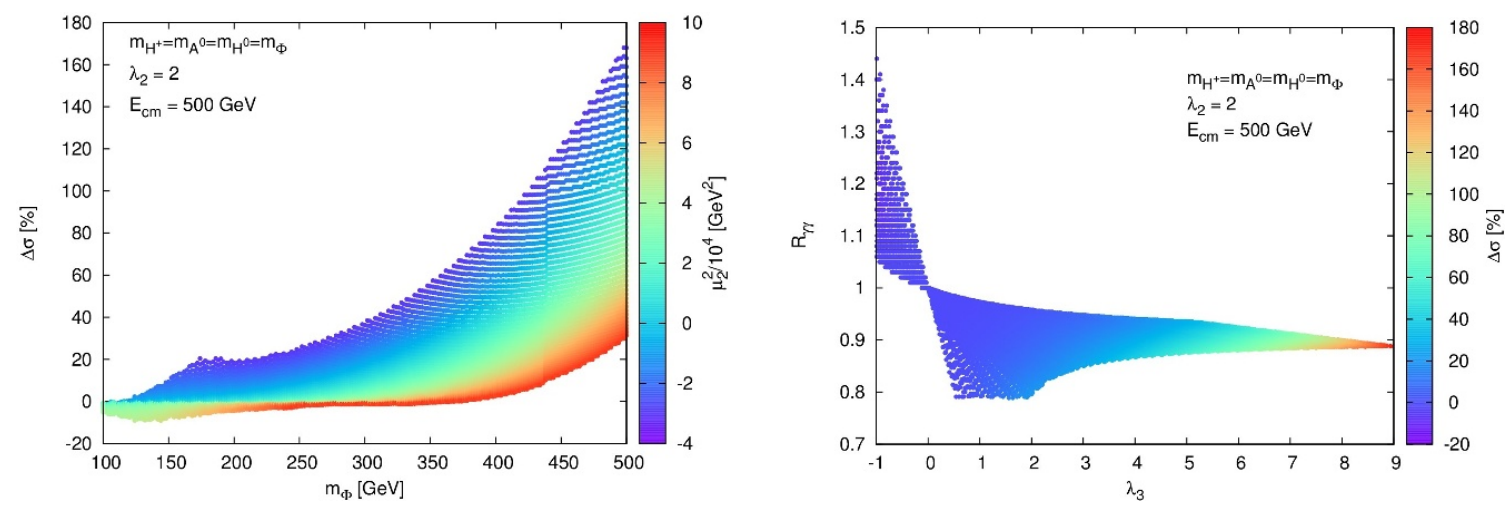

Figure 10. (Left): $\Delta \sigma$ as a function of $m_{\Phi}$ for $\sqrt{s}=500 \mathrm{GeV} \mu_{2}^{2}$ values are shown in the right column, (right) $R_{\gamma \gamma}$ as a function of $\lambda_{3}$ and the right column shows $\Delta \sigma$ in $\%$.

This explains that in most of the case, the corrections to the cross section are positive and confirm that the behavior of the $\Delta \sigma$ is consistent with our analysis concerning the trilinear Higgs self coupling in the IHDM. We stress that the enhancement of $\Delta \sigma$ is observed in a large part of the parameter space and can exceed $100 \%$ only in the high mass region $m_{\Phi} \geq 400 \mathrm{GeV}$ for $\mu_{2}^{2}<0$.

We plot in figure 10 (right) the ratio $R_{\gamma \gamma}$ as a function of $\lambda_{3}$ and showing the relative corrections in the left column. For small values of $-1<\lambda_{3}<2$ (low $m_{\Phi}$ ) the corrections are quite small. For $\lambda_{3}>4$ (high $m_{\Phi}$ ), $\Delta \sigma$ becomes more important and exceed $100 \%$, this region corresponds to $R_{\gamma \gamma} \approx 0.9 \pm 0.02$. Note that our results concerning $R_{\gamma \gamma}$ are in agreement with the results of [42].

\section{Conclusion}

We have computed the radiative corrections to triple Higgs coupling $h h h, h Z Z$ coupling as well as $e^{+} e^{-} \rightarrow Z h h$ in the framework of inert Higgs doublet model taking into account theoretical and experimental constraint on the parameter space of the model. The calculation was done in the Feynman gauge using dimensional regularization in the on-shell scheme. In the SM it is known that the top contribution to hhh coupling is of the order $10 \%$, we found that the bosonic contribution is somehow significant and goes up to $5 \%$. In the IHDM, we found that the total radiative corrections to the triple Higgs coupling could be substantial exceeding $100 \%$ for heavy dark Higgs masses $m_{H}, m_{A}$ and $m_{H \pm}$. We also show that the corrections to $h h h$ are decoupling for large $m_{\Phi}$ and large $\mu_{2}^{2}$. In the case of $h Z Z$ coupling the effect is rather mild and do not exceed $2.5 \%$. We also evaluate radiative corrections to the double Higgs Strahlung process $e^{+} e^{-} \rightarrow Z h h$ by looking only to the correction to the diagram that contribute to the signal i.e. the triple coupling $h h h$. We have shown that the correction are also very important. In general, the size of the loop effects, typically large, makes their proper inclusion in phenomenological analyses for future $e^{+} e^{-} \mathrm{LC}$ indispensable. 


\section{A The triple Higgs coupling in the IHDM: analytical results}

In this appendix, we present the analytical expression of the Higgs triple coupling at the one-loop order with the contribution of the inert scalars only. We use the Feynman diagrammatic method. The Feynman diagrams contributing to this process are shown in figure 1. By using the dimensional regularization, the amplitude is given by:

$$
\begin{aligned}
\Gamma_{h h h}^{\text {loop }}\left(q^{2}, m_{\Phi}^{2}\right)= & \frac{\lambda_{3}^{2} m_{W} s_{W}}{8 e \pi^{2}}\left(B_{0}\left(q^{2}, m_{H \pm}^{2}, m_{H \pm}^{2}\right)+2 B_{0}\left(m_{h}^{2}, m_{H \pm}^{2}, m_{H \pm}\right)\right. \\
& \left.+\frac{2 \lambda_{3} m_{W}^{2} s_{W}^{2}}{\pi \alpha} C_{0}\left(q^{2}, m_{h}^{2}, m_{h}^{2}, m_{H \pm}^{2}, m_{H \pm}^{2}, m_{H \pm}^{2}\right)\right) \\
& +\frac{\left(\lambda_{3}+\lambda_{4}+\lambda_{5}\right)^{2} m_{W} s_{W}}{16 e \pi^{2}}\left(B_{0}\left(q^{2}, m_{H^{0}}^{2}, m_{H^{0}}^{2}\right)+2 B_{0}\left(m_{h}^{2}, m_{H^{0}}^{2}, m_{H^{0}}^{2}\right)\right. \\
& \left.\times \frac{2\left(\lambda_{3}+\lambda_{4}+\lambda_{5}\right) m_{W}^{2} s_{W}^{2}}{\pi \alpha} C_{0}\left(q^{2}, m_{h}^{2}, m_{h}^{2}, m_{H^{0}}^{2}, m_{H^{0}}^{2}, m_{H^{0}}^{2}\right)\right) \\
& +\frac{\left(\lambda_{3}+\lambda_{4}-\lambda_{5}\right)^{2} m_{W} s_{W}}{16 e \pi^{2}}\left(B_{0}\left(q^{2}, m_{A^{0}}^{2}, m_{A^{0}}^{2}\right)+2 B_{0}\left(m_{h}^{2}, m_{A^{0}}^{2}, m_{A^{0}}^{2}\right)\right. \\
& \left.\times \frac{2\left(\lambda_{3}+\lambda_{4}-\lambda_{5}\right) m_{W}^{2} s_{W}^{2}}{\pi \alpha} C_{0}\left(q^{2}, m_{h}^{2}, m_{h}^{2}, m_{A^{0}}^{2}, m_{A^{0}}^{2}, m_{A^{0}}^{2}\right)\right)
\end{aligned}
$$

Where $B_{0}$ and $C_{0}$ are the Passarino-Veltman functions [71-73].

Following the on-shell renormalization scheme, there are six renormalization constants to compute: $\delta m_{h}^{2}, \delta m_{W}^{2}, \delta m_{Z}^{2}, \delta t, \delta Z_{e}, \delta Z_{h}$ and $\delta s_{W} . \delta Z_{A A}$ and $\delta Z_{Z A}$ are the field renormalization constant for the photon and $Z-\gamma$ mixing respectively and are given by:

$$
\begin{aligned}
\delta Z_{A A} & =\left.\frac{\alpha}{\pi} \frac{\partial}{\partial p^{2}} B_{00}\left(p^{2}, m_{H^{ \pm}}^{2}, m_{H^{ \pm}}^{2}\right)\right|_{p^{2}=0}, \\
\delta Z_{Z A} & =\frac{\alpha\left(1-2 s_{W}^{2}\right)}{2 m_{Z^{2}}^{2} c_{W} \pi}\left(-A_{0}\left(m_{H^{ \pm}}^{2}\right)+2 B_{00}\left(0, m_{H^{ \pm}}^{2}, m_{H^{ \pm}}^{2}\right)\right) .
\end{aligned}
$$

The electric charge renormalization constant as well as the renormalization constants for the $W$ and $Z$ masses are given by:

$$
\begin{aligned}
\delta Z_{e}= & -\frac{1}{2}\left(\delta Z_{A A}-\frac{s_{W}}{c_{W}} \delta Z_{Z A}\right), \\
\delta m_{W}^{2}= & \frac{\alpha}{16 \pi s_{W}^{2}}\left(2 A_{0}\left(m_{H^{ \pm}}^{2}\right)+A_{0}\left(m_{A^{0}}^{2}\right)+A_{0}\left(m_{H^{0}}^{2}\right)+4 B_{00}\left(m_{W}^{2}, m_{H^{ \pm}}^{2}, m_{H^{0}}^{2}\right)\right) \\
\delta m_{Z}^{2}= & -\frac{\alpha}{16 \pi s_{W}^{2} c_{W}^{2}}\left(-A_{0}\left(m_{A^{0}}^{2}\right)-A_{0}\left(m_{H^{0}}^{2}\right)-2\left(1-2 s_{W}^{2}\right)^{2} A_{0}\left(m_{H^{ \pm}}^{2}\right)+4 B_{00}\left(m_{Z}^{2}, m_{H^{0}}^{2}, m_{A^{0}}^{2}\right)\right. \\
& \left.+4\left(1-2 s_{W}^{2}\right)^{2} B_{00}\left(m_{Z}^{2}, m_{H^{ \pm}}^{2}, m_{H^{ \pm}}^{2}\right)\right) .
\end{aligned}
$$

The counter term $\delta s_{W}$, corresponding to the Weinberg mixing angle, is determined from the tree level relation $s_{W}^{2}=1-m_{W}^{2} / m_{Z}^{2} . \delta s_{W}$ is given by:

$$
\delta s_{W}=\frac{-c_{W}^{2}}{2 s_{W}}\left(\frac{\delta m_{W}^{2}}{m_{W}^{2}}-\frac{\delta m_{Z}^{2}}{m_{Z}^{2}}\right) .
$$


The counter-terms for the Higgs mass, Higgs field and the tadpole are given by:

$$
\begin{aligned}
\delta m_{h}^{2}= & \frac{1}{32 \pi}\left(2 \lambda_{3} A_{0}\left(m_{H^{ \pm}}^{2}\right)+\left(\lambda_{3}+\lambda_{4}+\lambda_{5}\right) A_{0}\left(m_{H^{0}}^{2}\right)+\left(\lambda_{3}+\lambda_{4}-\lambda_{5}\right) A_{0}\left(m_{A^{0}}^{2}\right)\right) \\
& +\frac{m_{W}^{2} s_{W}^{2}}{32 \alpha \pi^{3}}\left(2 \lambda_{3}^{2} B_{0}\left(m_{h}^{2}, m_{H^{ \pm}}^{2}, m_{H^{ \pm}}^{2}\right)+\left(\lambda_{3}+\lambda_{4}+\lambda_{5}\right)^{2} B_{0}\left(m_{h}^{2}, m_{H^{0}}^{2}, m_{H^{0}}^{2}\right)\right. \\
& \left.+\left(\lambda_{3}+\lambda_{4}-\lambda_{5}\right)^{2} B_{0}\left(m_{h}^{2}, m_{A^{0}}^{2}, m_{A^{0}}^{2}\right)\right) \\
\delta Z_{h}= & \frac{m_{W}^{2} s_{W}^{2}}{32 \alpha \pi^{3}}\left(2 \lambda_{3}^{2} \frac{\partial}{\partial p^{2}} B_{0}\left(p^{2}, m_{H^{ \pm}}^{2}, m_{H^{ \pm}}^{2}\right)+\left(\lambda_{3}+\lambda_{4}+\lambda_{5}\right)^{2} \frac{\partial}{\partial p^{2}} B_{0}\left(p^{2}, m_{H^{0}}^{2}, m_{H^{0}}^{2}\right)\right. \\
& \left.+\left(\lambda_{3}+\lambda_{4}-\lambda_{5}\right)^{2} \frac{\partial}{\partial p^{2}} B_{0}\left(p^{2}, m_{A^{0}}^{2}, m_{A^{0}}^{2}\right)\right)\left.\right|_{p^{2}=m_{h}^{2}} \\
\delta t= & -\frac{m_{W} s_{W}}{16 e \pi^{2}} \frac{1}{32 \pi}\left(2 \lambda_{3} A_{0}\left(m_{H^{ \pm}}^{2}\right)+\left(\lambda_{3}+\lambda_{4}+\lambda_{5}\right) A_{0}\left(m_{H^{0}}^{2}\right)+\left(\lambda_{3}+\lambda_{4}-\lambda_{5}\right) A_{0}\left(m_{A^{0}}^{2}\right)\right) .
\end{aligned}
$$

The expression for the triple Higgs coupling counter term is given in the third section eq. (3.7). The renormalized triple Higgs coupling is given by:

$$
\widetilde{\Gamma}_{h h h}^{\text {loop }}=\Gamma_{h h h}^{\text {loop }}+\delta \Gamma_{h h h}^{\text {loop }} .
$$

We have checked that the renormalized amplitude is UV-finite and furthermore independent of the renormalization scale $\mu$. We derive an approximate formula for $\widetilde{\Gamma}_{h h h}^{\text {loop }}$ In the limit $m_{\phi}=m_{H}=m_{A^{0}}=m_{H^{ \pm}}$and find a good agreement with full expression.

$$
\begin{aligned}
& \widetilde{\Gamma}_{h h h}^{\text {loop }}\left(q^{2}, m_{h}^{2}, m_{\phi}^{2}\right) \approx \\
& \frac{1}{8 \alpha e \pi^{3} q^{2}}\left[3 \alpha \pi q^{2} m_{W} s_{W}\left(2 \lambda_{3}^{2}+2 \lambda_{3} \lambda_{4}+\lambda_{4}^{2}+\lambda_{5}^{2}\right)\left(x_{1} \log \left(\frac{-1+x_{1}}{x_{1}}\right)+x_{2} \log \left(\frac{-1+x_{2}}{x_{2}}\right)\right)\right. \\
& \left.+\left(2 \lambda_{3}^{3}+3 \lambda_{3}^{2} \lambda_{4}+3 \lambda_{3} \lambda_{4}^{2}+\lambda_{4}^{3}+3\left(\lambda_{3}+\lambda_{4}\right) \lambda_{5}^{2}\right) m_{W}^{2} s_{W}^{2} \log ^{2}\left(-\frac{q^{2}}{m_{\phi}^{2}}\right)\right] \\
& +\frac{x_{1} x_{2}}{64 \alpha m_{W}^{3} m_{Z}^{2} \pi^{3} s_{W}^{5}\left(x_{1}-x_{2}\right)}\left(\left(2 \lambda_{3}^{2}+2 \lambda_{3} \lambda_{4}+\lambda_{4}^{2}+\lambda_{5}^{2}\right)\left(15 e m_{W}^{4} m_{Z}^{2}\right)\right. \\
& +4 e \alpha^{2} m_{h}^{2} \pi^{2}\left(m_{Z}^{2}\left(m_{h}^{2}-2 m_{W}^{2}+m_{Z}^{2}\right)\left(-1+2 s_{W}^{2}\right)+m_{\phi}^{2}\left(-2 m_{Z}^{2}+m_{W}^{2}\left(1+\left(c_{W}^{2}-s_{W}^{2}\right)^{2}\right)\right.\right. \\
& \left.\left.\left.+4 m_{Z}^{2} s_{W}^{2}\right)\right)\right) \\
& \times\left(\left(-1+x_{2}\right) \log \left(\frac{-1+x_{2}}{x_{2}}\right)+\left(1-x_{1}\right) \log \left(\frac{-1+x_{1}}{x_{1}}\right)\right) \\
& +\frac{x_{1} x_{2}}{64 m_{W}^{3} \pi s_{W}^{5}\left(x_{1}-x_{2}\right)}\left(\alpha e m _ { h } ^ { 2 } \left(-2 m_{Z}^{2}+m_{W}^{2}\left(3+\left(c_{W}^{2}-s_{W}^{2}\right)^{2}-4 s_{W}^{2}\right)\right.\right. \\
& \left.\left.+4 m_{Z}^{2} s_{W}^{2}+m_{h}^{2}\left(-2+4 s_{W}^{2}\right)\right)\right)
\end{aligned}
$$




$$
\begin{aligned}
& \times\left(x_{1} \log \left(\frac{-1+x_{2}}{x_{2}}\right)-x_{2} \log \left(\frac{-1+x_{1}}{x_{1}}\right)\right) \\
& +\frac{\alpha^{2} m_{h}^{2} m_{Z}^{2} \pi^{2}}{16 e m_{W}^{3} m_{Z}^{2} \pi^{2} s_{W}^{5}}\left[-2 m_{Z}^{2}+m_{W}^{2}\left(3+\left(c_{W}^{2}-s_{W}^{2}\right)^{2}-4 s_{W}^{2}\right)+4 m_{Z}^{2} s_{W}^{2}+m_{h}^{2}\left(-2+4 s_{W}^{2}\right)\right] \\
& \times\left(x_{1}^{3} \log \left(\frac{-1+x_{1}}{x_{1}}\right)-x_{2}^{3} \log \left(\frac{-1+x_{2}}{x_{2}}\right)\right)
\end{aligned}
$$

where $x_{1,2}$ are given by:

$$
x_{1,2}=\frac{1 \mp \sqrt{1-4 m_{\phi}^{2} / q^{2}}}{2} .
$$

\section{Acknowledgments}

A.A. would like to thank NCTS for financial support where part of this work has been done. This work was supported by the Moroccan Ministry of Higher Education and Scientific Research MESRSFC and CNRST: "Projet dans les domaines prioritaires de la recherche scientifique et du développement technologique": PPR/2015/6. A.J. would like to thank ICTP-Trieste for financial support during his stay where part of this work has been done.

Open Access. This article is distributed under the terms of the Creative Commons Attribution License (CC-BY 4.0), which permits any use, distribution and reproduction in any medium, provided the original author(s) and source are credited.

\section{References}

[1] F. Englert and R. Brout, Broken Symmetry and the Mass of Gauge Vector Mesons, Phys. Rev. Lett. 13 (1964) 321 [INSPIRE].

[2] P.W. Higgs, Broken Symmetries and the Masses of Gauge Bosons, Phys. Rev. Lett. 13 (1964) 508 [INSPIRE].

[3] P.W. Higgs, Broken symmetries, massless particles and gauge fields, Phys. Lett. 12 (1964) 132 [INSPIRE].

[4] P.W. Higgs, Spontaneous Symmetry Breakdown without Massless Bosons, Phys. Rev. 145 (1966) 1156 [INSPIRE].

[5] ATLAS collaboration, Observation of a new particle in the search for the Standard Model Higgs boson with the ATLAS detector at the LHC, Phys. Lett. B 716 (2012) 1 [arXiv: 1207.7214] [INSPIRE].

[6] ATLAS collaboration, Evidence for Higgs Boson Decays to the $\tau^{+} \tau^{-}$Final State with the ATLAS Detector, ATLAS-CONF-2013-108 (2013) [INSPIRE].

[7] ATLAS collaboration, Search for the bb decay of the Standard Model Higgs boson in associated W/ZH production with the ATLAS detector, ATLAS-CONF-2013-079 (2013) [INSPIRE].

[8] CMS collaboration, Observation of a new boson at a mass of $125 \mathrm{GeV}$ with the CMS experiment at the LHC, Phys. Lett. B 716 (2012) 30 [arXiv:1207.7235] [INSPIRE]. 
[9] CMS collaboration, Search for the standard model Higgs boson produced in association with a W or a Z boson and decaying to bottom quarks, Phys. Rev. D 89 (2014) 012003 [arXiv: 1310.3687] [INSPIRE].

[10] CMS collaboration, Evidence for the $125 \mathrm{GeV}$ Higgs boson decaying to a pair of $\tau$ leptons, JHEP 05 (2014) 104 [arXiv: 1401.5041] [INSPIRE].

[11] E.W.N. Glover and J.J. van der Bij, Higgs Boson Pair Production Via Gluon Fusion, Nucl. Phys. B 309 (1988) 282 [inSPIRE].

[12] C.-T. Lu, J. Chang, K. Cheung and J.S. Lee, An exploratory study of Higgs-boson pair production, JHEP 08 (2015) 133 [arXiv: 1505.00957] [INSPIRE].

[13] T. Plehn, M. Spira and P.M. Zerwas, Pair production of neutral Higgs particles in gluon-gluon collisions, Nucl. Phys. B 479 (1996) 46 [Erratum ibid. B 531 (1998) 655] [hep-ph/9603205] [INSPIRE].

[14] J. Baglio, A. Djouadi, R. Gröber, M.M. Mühlleitner, J. Quevillon and M. Spira, The measurement of the Higgs self-coupling at the LHC: theoretical status, JHEP 04 (2013) 151 [arXiv: 1212.5581] [INSPIRE].

[15] U. Baur, T. Plehn and D.L. Rainwater, Probing the Higgs selfcoupling at hadron colliders using rare decays, Phys. Rev. D 69 (2004) 053004 [hep-ph/0310056] [INSPIRE].

[16] A. Djouadi, W. Kilian, M.M. Mühlleitner and P.M. Zerwas, Production of neutral Higgs boson pairs at LHC, Eur. Phys. J. C 10 (1999) 45 [hep-ph/9904287] [inSPIRE].

[17] M.J. Dolan, C. Englert and M. Spannowsky, Higgs self-coupling measurements at the LHC, JHEP 10 (2012) 112 [arXiv:1206.5001] [INSPIRE].

[18] A. Papaefstathiou, L.L. Yang and J. Zurita, Higgs boson pair production at the LHC in the $b \bar{b} W^{+} W^{-}$channel, Phys. Rev. D 87 (2013) 011301 [arXiv:1209.1489] [InSPIRE].

[19] A. Ahriche, A. Arhrib and S. Nasri, Triple Higgs Coupling as a Probe of the Twin-Peak Scenario, Phys. Lett. B 743 (2015) 279 [arXiv: 1407.5283] [INSPIRE].

[20] A. Arhrib, R. Benbrik, C.-H. Chen, R. Guedes and R. Santos, Double Neutral Higgs production in the Two-Higgs doublet model at the LHC, JHEP 08 (2009) 035 [arXiv: 0906.0387] [INSPIRE].

[21] D.M. Asner et al., ILC Higgs White Paper, arXiv:1310.0763 [inSPIRE].

[22] A. Djouadi, W. Kilian, M.M. Mühlleitner and P.M. Zerwas, Testing Higgs selfcouplings at $e^{+} e^{-}$linear colliders, Eur. Phys. J. C 10 (1999) 27 [hep-ph/9903229] [InSPIRE].

[23] F. Borzumati and E. Kato, The Higgs boson and the International Linear Collider, arXiv: 1407.2133 [INSPIRE].

[24] ILD collaboration, J. Tian and K. Fujii, Measurement of Higgs couplings and self-coupling at the ILC, PoS (EPS-HEP 2013) 316 [arXiv: 1311.6528] [INSPIRE].

[25] N.G. Deshpande and E. Ma, Pattern of Symmetry Breaking with Two Higgs Doublets, Phys. Rev. D 18 (1978) 2574 [inSPIRE].

[26] M. Gustafsson, E. Lundstrom, L. Bergstrom and J. Edsjo, Significant Gamma Lines from Inert Higgs Dark Matter, Phys. Rev. Lett. 99 (2007) 041301 [astro-ph/0703512] [INSPIRE].

[27] T. Hambye and M.H.G. Tytgat, Electroweak symmetry breaking induced by dark matter, Phys. Lett. B 659 (2008) 651 [arXiv:0707.0633] [INSPIRE]. 
[28] P. Agrawal, E.M. Dolle and C.A. Krenke, Signals of Inert Doublet Dark Matter in Neutrino Telescopes, Phys. Rev. D 79 (2009) 015015 [arXiv:0811.1798] [InSPIRE].

[29] E. Nezri, M.H.G. Tytgat and G. Vertongen, $e^{+}$and $\bar{p}$ from inert doublet model dark matter, JCAP 04 (2009) 014 [arXiv:0901.2556] [InSPIRE].

[30] E.M. Dolle and S. Su, The Inert Dark Matter, Phys. Rev. D 80 (2009) 055012 [arXiv:0906.1609] [INSPIRE].

[31] A. Carmona and M. Chala, Composite Dark Sectors, JHEP 06 (2015) 105 [arXiv: 1504.00332] [INSPIRE].

[32] A. Arhrib, Y.-L.S. Tsai, Q. Yuan and T.-C. Yuan, An Updated Analysis of Inert Higgs Doublet Model in light of the Recent Results from LUX, PLANCK, AMS-02 and LHC, JCAP 06 (2014) 030 [arXiv:1310.0358] [inSPIRE].

[33] E. Ma, Verifiable radiative seesaw mechanism of neutrino mass and dark matter, Phys. Rev. D 73 (2006) 077301 [hep-ph/0601225] [INSPIRE].

[34] S. Andreas, M.H.G. Tytgat and Q. Swillens, Neutrinos from Inert Doublet Dark Matter, JCAP 04 (2009) 004 [arXiv:0901.1750] [INSPIRE].

[35] R. Barbieri, L.J. Hall and V.S. Rychkov, Improved naturalness with a heavy Higgs: An Alternative road to LHC physics, Phys. Rev. D 74 (2006) 015007 [hep-ph/0603188] [INSPIRE].

[36] A. Arhrib, R. Benbrik and T.-C. Yuan, Associated Production of Higgs at Linear Collider in the Inert Higgs Doublet Model, Eur. Phys. J. C 74 (2014) 2892 [arXiv:1401.6698] [INSPIRE].

[37] A. Goudelis, B. Herrmann and O. Stål, Dark matter in the Inert Doublet Model after the discovery of a Higgs-like boson at the LHC, JHEP 09 (2013) 106 [arXiv:1303.3010] [INSPIRE].

[38] A.G. Akeroyd, A. Arhrib and E.-M. Naimi, Note on tree level unitarity in the general two Higgs doublet model, Phys. Lett. B 490 (2000) 119 [hep-ph/0006035] [INSPIRE].

[39] I.F. Ginzburg, K.A. Kanishev, M. Krawczyk and D. Sokolowska, Evolution of Universe to the present inert phase, Phys. Rev. D 82 (2010) 123533 [arXiv: 1009.4593] [INSPIRE].

[40] M.E. Peskin and T. Takeuchi, Estimation of oblique electroweak corrections, Phys. Rev. D 46 (1992) 381 [INSPIRE].

[41] GFitTer Group collaboration, M. Baak et al., The global electroweak fit at NNLO and prospects for the LHC and ILC, Eur. Phys. J. C 74 (2014) 3046 [arXiv:1407.3792] [INSPIRE].

[42] A. Arhrib, R. Benbrik and N. Gaur, $H \rightarrow \gamma \gamma$ in Inert Higgs Doublet Model, Phys. Rev. D 85 (2012) 095021 [arXiv:1201.2644] [INSPIRE].

[43] G. Bélanger, B. Dumont, A. Goudelis, B. Herrmann, S. Kraml and D. Sengupta, Dilepton constraints in the Inert Doublet Model from Run 1 of the LHC, Phys. Rev. D 91 (2015) 115011 [arXiv: 1503.07367] [INSPIRE].

[44] E. Dolle, X. Miao, S. Su and B. Thomas, Dilepton Signals in the Inert Doublet Model, Phys. Rev. D 81 (2010) 035003 [arXiv:0909.3094] [INSPIRE].

[45] X. Miao, S. Su and B. Thomas, Trilepton Signals in the Inert Doublet Model, Phys. Rev. D 82 (2010) 035009 [arXiv: 1005.0090] [InSPIRE]. 
[46] M. Gustafsson, S. Rydbeck, L. Lopez-Honorez and E. Lundstrom, Status of the Inert Doublet Model and the Role of multileptons at the LHC, Phys. Rev. D 86 (2012) 075019 [arXiv: 1206.6316] [INSPIRE].

[47] CMS collaboration, Precise determination of the mass of the Higgs boson and studies of the compatibility of its couplings with the standard model, CMS-PAS-HIG-14-009 (2014) [INSPIRE].

[48] ATLAS collaboration, Measurement of Higgs boson production in the diphoton decay channel in pp collisions at center-of-mass energies of 7 and $8 \mathrm{TeV}$ with the ATLAS detector, Phys. Rev. D 90 (2014) 112015 [arXiv:1408.7084] [InSPIRE].

[49] B.A. Kniehl, Radiative corrections for $H \rightarrow Z Z$ in the standard model, Nucl. Phys. B 352 (1991) 1 [INSPIRE].

[50] S. Kanemura, Y. Okada, E. Senaha and C.P. Yuan, Higgs coupling constants as a probe of new physics, Phys. Rev. D 70 (2004) 115002 [hep-ph/0408364] [INSPIRE].

[51] S. Kanemura, S. Kiyoura, Y. Okada, E. Senaha and C.P. Yuan, New physics effect on the Higgs selfcoupling, Phys. Lett. B 558 (2003) 157 [hep-ph/0211308] [INSPIRE].

[52] S. Kanemura, M. Kikuchi and K. Yagyu, Fingerprinting the extended Higgs sector using one-loop corrected Higgs boson couplings and future precision measurements, Nucl. Phys. B 896 (2015) 80 [arXiv:1502.07716] [INSPIRE].

[53] W. Hollik and S. Penaranda, Yukawa coupling quantum corrections to the selfcouplings of the lightest MSSM Higgs boson, Eur. Phys. J. C 23 (2002) 163 [hep-ph/0108245] [INSPIRE].

[54] A. Dobado, M.J. Herrero, W. Hollik and S. Penaranda, Selfinteractions of the lightest MSSM Higgs boson in the large pseudoscalar mass limit, Phys. Rev. D 66 (2002) 095016 [hep-ph/0208014] [INSPIRE].

[55] T. Hahn, Generating Feynman diagrams and amplitudes with FeynArts 3, Comput. Phys. Commun. 140 (2001) 418 [hep-ph/0012260] [INSPIRE].

[56] T. Hahn and C. Schappacher, The Implementation of the minimal supersymmetric standard model in FeynArts and FormCalc, Comput. Phys. Commun. 143 (2002) 54 [hep-ph/0105349] [INSPIRE].

[57] T. Hahn and M. Pérez-Victoria, Automatized one loop calculations in four-dimensions and D-dimensions, Comput. Phys. Commun. 118 (1999) 153 [hep-ph/9807565] [INSPIRE].

[58] J. Küblbeck, M. Böhm and A. Denner, Feyn Arts: Computer Algebraic Generation of Feynman Graphs and Amplitudes, Comput. Phys. Commun. 60 (1990) 165 [INSPIRE].

[59] G.J. van Oldenborgh, FF: A Package to evaluate one loop Feynman diagrams, Comput. Phys. Commun. 66 (1991) 1 [inSPIRE].

[60] T. Hahn, Loop calculations with FeynArts, FormCalc and LoopTools, Acta Phys. Polon. B 30 (1999) 3469 [hep-ph/9910227] [INSPIRE].

[61] T. Hahn, Feynman Diagram Calculations with FeynArts, FormCalc and LoopTools, PoS (ACAT2010) 078 [arXiv: 1006. 2231] [INSPIRE].

[62] M. Böhm, H. Spiesberger and W. Hollik, On the One Loop Renormalization of the Electroweak Standard Model and Its Application to Leptonic Processes, Fortsch. Phys. 34 (1986) 687 [INSPIRE]. 
[63] A. Denner, Techniques for calculation of electroweak radiative corrections at the one loop level and results for W physics at LEP-200, Fortsch. Phys. 41 (1993) 307 [arXiv:0709.1075] [INSPIRE].

[64] K. Cheung, J.S. Lee and P.-Y. Tseng, Higgs Precision (Higgcision) Era begins, JHEP 05 (2013) 134 [arXiv: 1302.3794] [INSPIRE].

[65] G. Bélanger, B. Dumont, U. Ellwanger, J.F. Gunion and S. Kraml, Status of invisible Higgs decays, Phys. Lett. B 723 (2013) 340 [arXiv:1302.5694] [INSPIRE].

[66] G. Bélanger, B. Dumont, U. Ellwanger, J.F. Gunion and S. Kraml, Global fit to Higgs signal strengths and couplings and implications for extended Higgs sectors, Phys. Rev. D 88 (2013) 075008 [arXiv: 1306 . 2941] [INSPIRE].

[67] J.R. Espinosa, M.M. Mühlleitner, C. Grojean and M. Trott, Probing for Invisible Higgs Decays with Global Fits, JHEP 09 (2012) 126 [arXiv:1205.6790] [INSPIRE].

[68] O. Lebedev, H.M. Lee and Y. Mambrini, Vector Higgs-portal dark matter and the invisible Higgs, Phys. Lett. B 707 (2012) 570 [arXiv:1111.4482] [InSPIRE].

[69] C. Englert, M. Spannowsky and C. Wymant, Partially (in)visible Higgs decays at the LHC, Phys. Lett. B 718 (2013) 538 [arXiv:1209.0494] [INSPIRE].

[70] G. Bélanger et al., Full $\mathcal{O}(\alpha)$ electroweak corrections to double Higgs strahlung at the linear collider, Phys. Lett. B 576 (2003) 152 [hep-ph/0309010] [INSPIRE].

[71] G. 't Hooft and M.J.G. Veltman, Regularization and Renormalization of Gauge Fields, Nucl. Phys. B 44 (1972) 189 [InSPIRE].

[72] G. 't Hooft and M.J.G. Veltman, Scalar One Loop Integrals, Nucl. Phys. B 153 (1979) 365 [INSPIRE].

[73] G. Passarino and M.J.G. Veltman, One Loop Corrections for $e^{+} e^{-}$Annihilation Into $\mu^{+} \mu^{-}$ in the Weinberg Model, Nucl. Phys. B 160 (1979) 151 [INSPIRE]. 